\title{
Plantas medicinais no tratamento de ansiedade e depressão: Uma revisão
}

\author{
Medicinal plants in the treatment of anxiety and depression: A review \\ Plantas medicinales en el tratamiento de la ansiedad y la depresión: una revisión
}

Recebido: 28/11/2021 | Revisado: 03/12/2021 | Aceito: 23/12/2021 | Publicado: 11/01/2022

José Cândido da Silva Nobrega (in Memoriam)
ORCID: https://orcid.org/0000-0002-0976-3763
Universidade Federal de Campina Grande, Brasil
E-mail: jcandidosn @uol.com.br
Alan Vinícius de Araújo Batisa
ORCID: https://orcid.org/0000-0002-4613-777X
Universidade Federal de Campina Grande, Brasil
E-mail: alan.vinicius@ifce.edu.br
Osvaldo Soares da Silva
ORCID: https://orcid.org/0000-0002-4608-0638
Universidade Federal de Campina Grande, Brasil
E-mail: osvaldo@ccta.ufcg.edu.br
Verônica Cristian Soares de Belchior
ORCID: https:// orcid.org/0000-0003-2357-9599
Universidade Federal de Campina Grande, Brasil
E-mail: prof_veronicabelchior@ hotmail.com
Wendell de Almeida Lacerda
ORCID: https://orcid.org/0000-0002-9737-1013
Faculdades Integradas do Ceará, Brasil
E-mail: wendelllacerada@gmail.com
Sandra Maijane Soares de Belchior
ORCID: https://orcid.org/0000-0001-5807-2259
Universidade Federal de Campina Grande, Brasil
E-mail: sandrabelchiorfic@gmail.com

\section{Resumo}

Há uma grande diversidade de plantas no Brasil, e muitas delas, ao serem estudadas, apresentam elevado teor nutricional, presença de antioxidante, entre outras propriedades, podendo ter um potencial farmacológico significativo e consequentemente gerando aplicações em diversas áreas como saúde, cosmética e culinária. As plantas medicinais são usadas por grande parte da população mundial, como um recurso medicinal alternativo para o tratamento de diversas doenças, visto que em muitas comunidades, representam um recurso mais acessível em diversos sentidos em relação aos medicamentos alopáticos. Destaca-se a utilização das plantas medicinais para fins terapêuticos, em que muitas atividades biológicas conferidas às plantas foram demonstradas, dentre elas a ação no sistema nervoso central, sendo utilizadas para o tratamento e/ou prevenção de transtornos psicossociais como ansiedade e depressão. Sendo assim, o presente estudo tem como objetivo realizar uma revisão bibliográfica sobre as plantas medicinais mais utilizadas no tratamento da ansiedade e depressão. Verificou-se que nos tratamentos, as plantas medicinais e os fitoterápicos são vistos como uma alternativa viável para o tratamento dos quadros de ansiedade e depressão, visto que possuem menos efeitos colaterais. Sendo assim, a presente pesquisa revisou as espécies: Hypericum perforatum L.; Passiflora incarnata L.; Melissa Officinalis L.; Chamomilla recutita L.; Piper methysticum G.; Erythrina verna; Humulus lupulus L. e Crataegus oxyacantha L. Foi possível apresentar a efetividade de todas as espécies citadas, com as descrições encontradas em estudos da literatura.

Palavras-chave: Fitoterápicos; Doenças psicossociais; Tratamentos alternativos.

\begin{abstract}
There is a great diversity of plants in Brazil, and many of them, when studied, have high nutritional content, presence of antioxidant, among other properties, and may have a significant pharmacological potential and consequently generating applications in various areas such as health, cosmetics and cooking. Medicinal plants are used by a large part of the world's population as an alternative medicinal resource for the treatment of various diseases, since in many communities they represent a more accessible resource in various senses in relation to allopathic medicines. The use of medicinal plants for therapeutic purposes is highlighted, in which many biological activities conferred on plants were demonstrated, among them the action on the central nervous system, being used for the treatment and/or prevention of psychosocial disorders such as anxiety and depression. Thus, the present study aims to conduct a literature review on the medicinal plants most used in the treatment of anxiety and depression. It was found that in
\end{abstract}


treatments, medicinal plants and herbal medicines are seen as a viable alternative for the treatment of anxiety and depression, since they have fewer side effects. Thus, the present research reviewed the species: Hypericum perforatum L.; Passiflora incarnata L.; Melissa L. Officinalis; Chamomilla L. recutita; Piper G. methysticum; Erythrina verna; Humulus lupulus L. and Crataegus oxyacantha L. It was possible to present the effectiveness of all the species mentioned, with the descriptions found in studies in the literature.

Keywords: Herbal medicines; Psychosocial disease, Alternative treatments.

\begin{abstract}
Resumen
Hay una gran diversidad de plantas en Brasil, y muchas de ellas, cuando se estudian, tienen alto contenido nutricional, presencia de antioxidantes, entre otras propiedades, y pueden tener un potencial farmacológico significativo y, en consecuencia, generar aplicaciones en diversas áreas como la salud, la cosmética y la cocina. Las plantas medicinales son utilizadas por gran parte de la población mundial como un recurso medicinal alternativo para el tratamiento de diversas enfermedades, ya que en muchas comunidades representan un recurso más accesible en diversos sentidos en relación con los medicamentos alopáticos. Se destaca el uso de plantas medicinales con fines terapéuticos, en el que se demostraron muchas actividades biológicas conferidas a las plantas, entre ellas la acción sobre el sistema nervioso central, siendo utilizadas para el tratamiento y/o prevención de trastornos psicosociales como la ansiedad y la depresión. Así, el presente estudio tiene como objetivo realizar una revisión bibliográfica sobre las plantas medicinales más utilizadas en el tratamiento de la ansiedad y la depresión. Se encontró que, en los tratamientos, las plantas y las hierbas medicinales son vistas como una alternativa viable para el tratamiento de la ansiedad y la depresión, ya que tienen menos efectos secundarios. Así, la presente investigación revisó las especies: Hypericum perforatum L.; Passiflora incarnata L.; Melissa L. Officinalis; Chamomilla L. recutita; Piper G. methysticum; Erythrina verna; Humulus lupulus L. y Crataegus oxyacantha L. Fue posible presentar la efectividad de todas las especies mencionadas, con las descripciones encontradas en estudios en la literatura.
\end{abstract}

Palabras clave: Hierbas medicinales; Enfermedades psicosociales; Tratamientos alternativos.

\title{
1. Introdução
}

O homem sempre teve a necessidade de lidar com o surgimento de sintomas no seu cotidiano, devido a traumatismos, uso de novos alimentos ou adoecimento. Sendo assim, empregava-se de plantas medicinais, conhecimento obtido a partir de experiências ou pela observação de sua utilização pelos animais (Mattos et al., 2018). Mesmo com o desenvolvimento dos fármacos sintéticos a partir do desenvolvimento tecnológico, as plantas medicinais continuaram como forma alternativa de tratamento em diversos países do mundo, observando-se nas últimas décadas a valorização do emprego de preparações à base de plantas para fins terapêuticos (Badke et al., 2012).

Hoje em dia, as plantas medicinais são usadas por grande parte da população mundial, como um recurso medicinal alternativo para o tratamento de diversas doenças, visto que em muitas comunidades, representam um recurso mais acessível em diversos sentidos em relação aos medicamentos alopáticos (Caneiro et al., 2014).

A ciência responsável pelo estudo das plantas é a fitoterapia, no qual trata dos métodos de tratamento caracterizado pela utilização de plantas medicinais em suas várias preparações, formando uma modalidade de terapia integrativa e complementar diante das necessidades de saúde (Oliveira et al., 2018). O avanço da fitoterapia se deu pela percepção da população sobre a utilização de plantas medicinais, no qual é visto como uma integrativa histórica à utilização de medicamentos sintéticos, tendo em vista que os químicos são considerados mais caros e agressivos ao organismo, bem como o baixo custo e fácil acesso à grande parcela da população brasileira (Santos et al., 2011).

As plantas medicinais são amplamente utilizadas no Brasil, visto que possui alguns facilitadores, como a grande diversidade ambiental e o baixo custo associado à terapêutica. Há uma grande diversidade de plantas em nosso país e, muitas delas, ao serem estudadas, apresentam elevado teor nutricional, presença de antioxidante, entre outras propriedades, podendo ter um potencial farmacológico significativo e, consequentemente, gerando aplicações em diversas áreas como saúde, cosmética, culinária (Castro \& Figueiredo, 2019).

Almeida et al. (2013) destacam que as pesquisas com plantas medicinais são crescentes pela capacidade dessas espécies de produzir moléculas com atividade terapêutica, empregadas para diversos fins terapêuticos, em que muitas 
atividades biológicas conferidas às plantas foram demonstradas, dentre elas a ação no sistema nervoso central, sendo utilizadas para o tratamento e/ou prevenção de transtornos psicossociais como ansiedade e depressão.

Os transtornos de ansiedade e depressão têm crescido de forma significativa nos últimos anos, principalmente pelo modo de vida moderna, podendo ter consequências importantes na adaptação social, tendo em vista que modificam a qualidade das relações interpessoais, e podem afetar o exercício das atividades pessoais e profissionais (Beltrami et al., 2013).

"A ansiedade é uma condição afetiva normal que, quando em excesso, pode acarretar distúrbios do humor, bem como de pensamento, de comportamento e da atividade fisiológica" (Almeida et al., 2011, p. 384). A ansiedade é definida por um estado psíquico de apreensão ou um medo exacerbado, instigado pela antecipação de uma situação desagradável, perigosa ou até mesmo em uma situação desafiadora. Os transtornos de ansiedade são comuns e resultam em grandes sofrimentos e importante comprometimento funcional (Cury, 2017).

Para Donelli et al., (2017) é uma vivência comum, potencialmente típica e experimentada e se caracteriza por um sentimento difuso, desagradável e vago de apreensão, como inquietação, desconforto e taquicardia. Ou seja, é uma resposta a uma ameaça desconhecida, interna, vaga ou de origem conflituosa. Do mesmo modo, essa vivência faz parte das experiências humanas e é um estado emocional esperado e necessário para a sobrevivência, visto que na sua ausência, o ser humano não lutaria, não procuraria vencer obstáculos e nem fugiria de situações perigosas.

Já a depressão, é uma desordem do humor que acomete mais da metade da população mundial e se caracteriza por sintomas debilitantes como "visões negativas do eu, do mundo e do mundo futuro, bem como recorrentes e incontroláveis pensamentos negativos que muitas vezes giram em torno do auto [...]" (Gotlib et al., 2010, p. 286). Para os autores, são três os principais mecanismos envolvidos no desenvolvimento da depressão: déficits na memória, respostas ruins a estados de humor negativos e a incapacidade de usar estímulos positivos e recompensadores para regular o humor negativo.

A depressão e ansiedade têm formas distintas de se manifestarem, entretanto, possuem fundamentos em comum, que são síndromes heterogêneas, de maneira suposta relacionadas devido a características da rotina, são fenômenos separados, os quais podem alternar-se ao longo do tempo (Carvalho et al., 2021).

Logo, destaca-se a utilização das plantas medicinais e os fitoterápicos dessas doenças psicossociais. Nesse sentido, o presente artigo tem como objetivo realizar uma revisão bibliográfica sobre as plantas medicinais mais utilizadas no tratamento da ansiedade e depressão.

\section{Metodologia}

O método de pesquisa é a revisão bibliográfica. Lakatos \& Marconi (2002, p. 71) afirmam que "a pesquisa bibliográfica não é mera repetição do que já foi dito ou escrito sobre certo assunto, mas propicia o exame de um tema sob novo enfoque ou abordagem, chegando a conclusões inovadoras".

Conforme caracteriza Köche (2016), do ponto de vista da natureza, esse trabalho trata de uma pesquisa básica. "Pesquisa básica é aquela dirigida à produção de conhecimentos fundamentais, i.e., a uma sólida fundamentação teórica sobre a qual futuras pesquisas poderão ser desenvolvidas, à compreensão de processos básicos humanos e naturais. A audiência primária da pesquisa básica são os pesquisadores da área básica" (Moreira \& Rizzatti, 2020). Ou seja, buscam responder perguntas para ampliar o conhecimento que temos a um relacionado tema. As pesquisas básicas envolvem verdades e interesses universais e tem como finalidade gerar novos conhecimentos para o avanço da ciência, mas sem aplicação prática.

Pela perspectiva de abordagem, é uma pesquisa qualitativa, ou seja, análises de dados indutivamente. Analisando os objetivos essa pesquisa é exploratória, isto é, seu objetivo é proporcionar maior familiaridade com o problema, tornar-se explicito ou construir hipóteses com seu respeito ou causar aprimoramento do tema (Pereira et al., 2018). 
Nesse sentido, o presente estudo foi realizado com base em uma revisão bibliográfica sistemática, utilizando trabalhos científicos acerca do tema, através da Biblioteca Virtual em Saúde - BVS, utilizando como pretensão de pesquisa as seguintes bases de dados LILACS e SCIELO.

A Biblioteca virtual em Saúde (BVS) e uma rede de informações online, coordenado pelo centro latino-americano de informações em ciências da saúde (BIREME), para compartilhamento científico e técnico em saúde, onde está disponível um acervo de bases de dados bibliográficos com o propósito de colaborar para o desenvolvimento da saúde, contribuindo para profissionais e estudantes com informações científicas (Biblioteca virtual em saúde, 2021).

LILACS (Literatura Latino Americana em Ciências de Saúde), e um índice bibliográfico de produção científica e técnica em ciências da saúde, onde são registrados documentos técnico-científicos das áreas da ciência em saúde, publicados na América latina e no caribe desde do ano de 1982, incluindo teses capítulos de livros artigos conferencias entre outros que estejam de acordo com o guia de seleção de documentos para a base dados LILACS (Biblioteca virtual em saúde, 2021).

SCIELO (Scientific Electronic Library Online) e uma biblioteca eletrônica que contém uma coleção de periódicos científicos com objetivo de desenvolver uma metodologias comum para a preparação e analises da produção cientifica, tem como parceiros FAPESP(Fundação de Amparo à Pesquisa do Estado de São Paulo) e com BIHEME(Centro Latino-Americano e do Caribe de Informação em Ciências da Saúde) e no ano de 2002 passou a contar com o apoio de outro conselho o CNPq (Conselho Nacional de Desenvolvimento Cientifico e Tecnológico) (Biblioteca virtual em saúde, 2021).

Como fatores de inclusão foram utilizados os dados obtidos através de publicações em revistas, artigos dentro dos últimos 10 anos, e artigos disponíveis na íntegra. Como fatores de exclusão: artigos que não contemplem o objetivo de pesquisa e em duplicidade. Conforme consulta nos Decs em Saúde, serão utilizados os seguintes descritores: "Plantas Medicinais"; " Fitoterapia"; "Depressão" e "Ansiedade", utilizando o operador booleano "and".

\section{Resultados e Discussão}

A coleta de dados para este trabalho teve início com a busca por artigos científicos, nas bases de dados descritas na metodologia, obedecendo-se os critérios de inclusão e exclusão estabelecidos previamente, o que permitiu verificar os quantitativos de trabalhos disponíveis à medida que as combinações de descritores formam utilizadas no mecanismo de busca.

O Quadro 1 apresenta esses quantitativos em função da combinação de descritores utilizadas.

Quadro 1: Quantitativos de artigos em função do descritor utilizado.

\begin{tabular}{|l|l|}
\hline \multicolumn{1}{|c|}{ DESCRITORES } & RESULTADOS \\
\hline (Plantas Medicinais) and (Fitoterapia) & 6015 artigos \\
\hline (Plantas Medicinais) and (Depressão) & 719 artigos \\
\hline (Plantas Medicinais) and (Ansiedade) & 249 artigos \\
\hline (Plantas Medicinais) and (Ansiedade) and (Depressão) & 95 artigos \\
\hline (Plantas Medicinais) and (Ansiedade) and (Fitoterapia) & 45 artigos \\
\hline (Plantas Medicinais) and (Ansiedade) and (Depressão) and (Fitoterapia) & 23 artigos \\
\hline
\end{tabular}

Fonte: Autores (2021). 
Assim, verifica-se uma grande diversidade de espécies que possuem potencialidade no tratamento de doenças mentais. O presente estudo revisou as espécies: Hypericum perforatum L.; Passiflora incarnata L.; Melissa Officinalis L.; Chamomilla recutita L.; Piper methysticum G.; Erythrina verna; Humulus lupulus L. e Crataegus oxyacantha L. O Quadro 2 apresenta os resultados da revisão bibliográfica.

Quadro 2: Plantas medicinais utilizadas no tratamento de ansiedade e depressão.

\begin{tabular}{|c|c|c|c|}
\hline Nome científico & Nome popular & Benefício & Autores \\
\hline Hypericum perforatum $\mathrm{L}$. & $\begin{array}{l}\text { Erva-de-são- } \\
\text { joão, hipérico } \\
\text { ou hipericão }\end{array}$ & $\begin{array}{l}\text { Combate a depressão leve a } \\
\text { moderada, bem como os sintomas } \\
\text { relacionados à ansiedade, tensão } \\
\text { muscular, déficit de atenção, } \\
\text { síndrome da fadiga crônica, } \\
\text { síndrome do intestino irritável e } \\
\text { TPM. }\end{array}$ & $\begin{array}{l}\text { Klemow et al. (2011) e } \\
\text { Alves (2014) }\end{array}$ \\
\hline Passiflora incarnata $\mathrm{L}$. & $\begin{array}{l}\text { Maracujá ou } \\
\text { Flor da paixão }\end{array}$ & $\begin{array}{l}\text { Possui propriedades sedativa, } \\
\text { calmante, sonífera e hipnótica. São } \\
\text { utilizadas no tratamento de } \\
\text { ansiedade, depressão, tensão } \\
\text { nervosa, insônia e déficit de } \\
\text { atenção. }\end{array}$ & Oliveira et al., (2020) \\
\hline Melissa Officinalis L. & Erva-cidreira & $\begin{array}{l}\text { Possui propriedade calmante, } \\
\text { sonífera, ansiedade, digestiva, } \\
\text { sedativa e antiespasmódica. }\end{array}$ & Cases et al. (2011) \\
\hline Chamomilla recutita $\mathrm{L}$. & Camomila & $\begin{array}{l}\text { Redução de sintomas do transtorno } \\
\text { de } \\
\text { ansiedade }\end{array}$ & Lima et al., (2019) \\
\hline Piper methysticum G. & Kava-kava & \begin{tabular}{|lrr} 
Tratamento & da & ansiedade, \\
agitação, & \multicolumn{2}{c}{ epilepsia, } \\
psicose, & depressão \\
distúrbios nervosos, como estresse \\
ou cansaço
\end{tabular} & Barbosa et al., (2013) \\
\hline Humulus lupulus $\mathrm{L}$. & $\begin{array}{l}\text { Lúpulo ou pé- } \\
\text { de-galo }\end{array}$ & $\begin{array}{l}\text { Alívio de insônia, excitação e } \\
\text { inquietação associadas a dores de } \\
\text { cabeça de tensão e nervosismo } \\
\text { tensão }\end{array}$ & Kyrou et al. (2017) \\
\hline Crataegus oxyacantha $L$. & $\begin{array}{l}\text { Espinheiro } \\
\text { branco } \\
\text { Pilriteiro }\end{array}$ & $\begin{array}{l}\text { Tratamento de ansiedade e insônia, } \\
\text { com propriedade relaxante. }\end{array}$ & Pereira et al. (2018) \\
\hline
\end{tabular}

Fonte: Autores (2021).

De acordo com Silva \& Silva (2021), o uso das plantas medicinais como recurso terapêutico no tratamento da ansiedade e depressão, vem apresentando uma opção viável em relação aos tratamentos com fármacos, tendo em vista que alguns pacientes não toleram os efeitos adversos ou não respondem aos tratamentos farmacológicos tradicionais.

Diversas espécies vegetais possuem propriedades terapêuticas, e, assim, o uso de plantas medicinais representa um fator essencial para a manutenção das condições de saúde das pessoas acometidas por doenças mentais (Bortoluzzie et al., 2020). Para Carvalho (2021), a utilização das plantas medicinais em casos de ansiedade e depressão vem aumentando em diversas partes do mundo, visto que possuem a mesma eficácia e menos risco de efeitos nocivos que os medicamentos convencionais.

Como afirmam Silva et al. (2021), existe uma variedade de fármacos, de diferentes classes terapêuticas, os quais apresentam eficácia no tratamento da ansiedade e depressão, no entanto, grande parte dessas substâncias apresentam reações indesejáveis como, por exemplo, dependência e síndrome de abstinência, aspectos estes que favorecem uma alta incidência de não adesão ao tratamento farmacológico. 
As principais espécies de plantas utilizadas no tratamento de ansiedade e depressão, e que, serão objetos de pesquisa desse estudo, são: Hypericum perforatum L.; Passiflora incarnata L.; Melissa Officinalis L.; Chamomilla recutita L.; Piper methysticum G.; Erythrina verna; Humulus lupulus L. e Crataegus oxyacantha L.

\section{Hypericum perforatum $L$.}

A espécie Hypericum perforatum L é conhecida popularmente como erva-de-são-joão, essa espécie pertence à família Guttiferae e possui em sua composição química hipericina e hiperforina, no qual possuem papel na atividade antidepressiva, apesar do mecanismo de ação não ser totalmente conhecido (Pavanelli, 2021).

A espécie Hypericum perforatum (hipérico) é um potente fitoterápico empregado no tratamento de múltiplas condições debilitantes do organismo, dentre as quais, a depressão. A planta é do gênero Hypericum, ao qual pertencem aproximadamente 400 espécies, sendo oriunda da Europa, Ásia e África. Devido à potencialidade de suas ações antibacterianas e anti-inflamatórias é empregada para tratamento de problemas dermatológicos, digestivos e psicológicos (Alves et al., 2014).

Existem evidências de que o extrato oriundo do hipérico seja tão eficaz quanto a classe de antidepressivos tricíclicos, mas não tem sua eficácia bem definida quando em comparação à classe de inibidores da receptação de serotonina (Zirak et al., 2019). Essa planta medicial pode ser uma alternativa apropriada para o tratamento sintomático de pacientes com depressão leve a moderada (Ng et al., 2017).

Alves et al. (2014) complementam ao afirmar que a planta contém ao menos dez classes de compostos biologicamente ativos, como a antraquinonas/naftodiantronas, derivados de floroglucinol, flavonoides, biflavonas, xantonas, óleos voláteis, aminoácidos, vitamina $\mathrm{C}$, cumarinas, taninos e carotenoides. Pela presença de flavonoides e hipericina, a planta possui atividade antioxidante que desempenha papel na atividade antidepressiva.

\section{Passiflora incarnata $\mathbf{L}$.}

O gênero Passiflora é conhecido popularmente por "maracujá" ou "flor da paixão" apresentando várias espécies vegetais com algum princípio fitoterapêutico, de forma especial para Passiflora incarnata L. que é largamente utilizada no tratamento dos sintomas de ansiedade, além de apresentar ações analgésica, antiespasmódica, antitérmica e anti-inflamatória. As moléculas que possibilitam essa planta possuir tantos efeitos benéficos para saúde mental, são: flavonoides e de saponinas, cumarinas, alcaloides, heterosídeos, fitoesteróides e de ácidos fenólicos (Oliveira et al., 2020).

De acordo com Lopes et al. (2017), essa planta possui propriedades terapêuticas pelo valor medicinal das folhas, em que o suco que contêm passiflorina, um sedativo natural e o chá preparado com as folhas têm efeito diurético e sedativo. Logo, os autores colaboram ao afirmar que a atuação da Passiflora incarnata no tratamento da ansiedade está associada com a inibição da monoamina oxidase e a ativação dos receptores de ácido gama-aminobutírico.

\section{Melissa Officinalis L.}

A espécie Melissa officinalis L. (mais conhecida popularmente como erva-cidreira), é amplamente utilizada no tratamento da ansiedade, bem como para tratar de crises nervosas, taquicardia, melancolia e histerismo (Jesus \& Oliveira, 2021). Os autores afirmam que se utiliza as folhas para se fazer chá, objetivando atuar como calmante, fazendo uma intervenção em casos de ansiedade e insônia. Além disso, pode ser utilizada como medicação para tratar a gripe, dispepsia, cefaleias, bronquite crônica, dores reumáticas, enxaqueca e doenças virais.

"A erva-cidreira possui uma grande quantidade de citral, limonemo, mirceno e carvona, apresentando ação comprovada contra dor, inflamação e atividade ansiolítica" (Silva et al., 2021, p. 5). Os autores corroboram ao afirmar que o citral possui ação calmante em consonância com limonemo e ação analgésica quando age com o mirceno. 
De acordo com Cases et al. (2011), A Melissa officinalis L. melhora o desempenho cognitivo e humor, reduz o estresse induzido e tem efeitos ansiolíticos nos seres humanos. Estes estudos, em que contém ácido rosmarínico e o triterpenoides pentacíclicos, ácidos ursólico e oleanólico que inibem o catabolismo do ácido gama-aminobutírico. Devido a sua eficácia em melhorar a ansiedade e pode ser uma alternativa às drogas farmacêuticas no tratamento da ansiedade.

\section{Chamomilla recutita $\mathbf{L}$.}

A camomila mostra-se eficaz no tratamento de diversas doenças como ação ansiolítica, antisséptico e carminativa. As atividades biológicas da camomila devem-se ao aparecimento de diversos conjuntos de elementos ativos, como os terpenos no qual exerce ação farmacológicas, bem como camazuleno e o bisabolol, em que são responsáveis pela ação anti-inflamatória e antiproliferativa (Amsterdam et al., 2020).

Essa espécie é amplamente utilizada tanto de forma empírica na medicina tradicional, como também na forma de medicamento fitoterápico, por meio da extração de flavonoides e óleo volátil presentes nos capítulos florais, responsáveis por suas propriedades terapêuticas, com destaque para a ação ansiolítica (Lima et al., 2019). Os pesquisadores complementam ao afirmar que seu mecanismo de ação ainda desconhecido, porém, acredita-se que os flavonóides podem produzir atividade ansiolítica afetando o GABA, noradrenalina (NA), dopamina (DA) e neurotransmissão de serotonina ou modulando a função do eixo hipotálamo-hipófise adrenocortical.

\section{Piper methysticum G.}

O Piper methysticum é um arbusto dióico, ereto, com 2 a 3 metros de altura, com folhas grandes e rígidas profundamente cortadas na base (Barbosa et al., 2013). Originada nas ilhas do Pacífico Sul, a Piper methysticum, é utilizada há anos para promover bem-estar, redução da fadiga e da ansiedade. Hoje em dia, tem sido utilizada como tratamento alternativo para distúrbios psiquiátricos, como o transtorno de ansiedade generalizada (Silva et al., 2021).

Barbosa et al. (2013) afirmam que o uso de $100 \mathrm{mg}$ dessa planta medicinal, na dose, três vezes ao dia, apresenta resultados positivos no tratamento da ansiedade, tensão, insônia e dores musculares, contribuindo também no tratamento de pacientes que apresentam sintomas de depressão, distimia, transtorno do pânico e outros distúrbios psiquiátricos.

\section{Humulus lupulus L.}

O Humulus lupulus L. tem uma longa tradição de uso medicinal na Europa. Lúpulo é o nome comum de os estrobilos (cones) das inflorescências femininas da planta Humulus lupulus L. e é largamente utilizada como um agente amargo na indústria cervejeira, como bem como um produto medicinal à base de ervas, principalmente para dormir distúrbios. Assim, o lúpulo sob a forma de chá de ervas, infusões, extratos líquidos/álcoólicos, tinturas e substâncias herbais em pó têm sido tradicionalmente usado para o alívio de insônia, excitação e inquietação associadas a dores de cabeça de tensão e nervosismo tensão (Kyrou et al., 2017).

\section{Crataegus oxyacantha $\mathbf{L}$.}

Crataegus oxyacantha L. é uma das duas espécies de espinheiro reconhecidas medicinalmente na Farmacopeia Europeia, no qual é conhecida popularmente como Espinheiro branco ou Pilriteiro, tem na sua composição flavonoides, procianidinas e aminas biogênicas que atuam no tratamento de ansiedade e insônia, com propriedade relaxante (Pereira et al., 2018).

Exerce várias atividades farmacológicas, como hipotensora, anti-hiperlipidêmica, anti-hiperglicêmica, ansiolítica, imunomoduladora e antimutagênica. Procianidinas oligoméricas e tipos de flavonóides flavonóides / flavonóides, que são 
considerados os principais grupos de substâncias ativas, ácidos fenólicos, triterpenos, ácidos graxos e esteróis estão presentes na planta (Orhan, 2018).

\section{Conclusão}

O presente artigo buscou realizar uma revisão bibliográfica sobre as plantas medicinais mais utilizadas no tratamento da ansiedade e depressão. As doenças mentais têm crescido de forma significativa nos últimos anos, especialmente pelo modo de vida moderna, podendo ter consequências importantes na qualidade de vida.

Nos tratamentos, destaca-se as plantas medicinais e os fitoterápicos, no qual são vistos como uma alternativa viável para o tratamento dos quadros de ansiedade e depressão, visto que possuem menos efeitos colaterais. Sendo assim, a presente pesquisa revisou as espécies: Hypericum perforatum L.; Passiflora incarnata L.; Melissa Officinalis L.; Chamomilla recutita L.; Piper methysticum G.; Erythrina verna; Humulus lupulus L. e Crataegus oxyacantha L. Foi possível apresentar a efetividade de todas as espécies citadas, com as descrições encontradas em estudos da literatura.

\section{Referências}

Almeida, A. A. F., Behlau, M. \& Leite, J. R. (2011). Correlação entre ansiedade e performance comunicativa. Revista da Sociedade Brasileira de Fonoaudiologia, 16, 384-389.

Almeida, A. A. C., Carvalho, R. B. F. d., Coelho, M. L., \& Freitas, R. M. d. (2013). Utilização de plantas medicinais para o tratamento da depressão: Uma prospecção tecnológica. Revista GEINTEC - Gestão, Invação e Tecnologias, 3(2), 157-166.

Alves, A. C. S., Moraes, D. C., De Freitas, G. B. L., \& Almeida, D. J. (2014). Aspectos botânicos, químicos, farmacológicos e terapêuticos do Hypericum perforatum L. Revista brasileira de plantas medicinais, 16, 593-606.

Amsterdam, J. D., Li, Q. S., Xie, S. X., \& Mao, J. J. (2020). Putative antidepressant effect of chamomile (Matricaria chamomilla L.) oral extract in subjects with comorbid generalized anxiety disorder and depression. The Journal of Alternative and Complementary Medicine, 26(9), 815-821.

Badke, M. R., Budó, M. L. D., Alvim, N. A. T. \& Zanetti, G. D. (2012). Saberes e práticas populares de cuidado em saúde com o uso de plantas medicinais. Texto \& Contexto-Enfermagem, 21, 363-370.

Barbosa, D. R., Lenardon, L., \& Partata, A. K. (2013). KAVA-KAVA (Piper methysticum): uma revisão geral. Revista Científica do ITPAC, 6(3), p. 1- 9.

Biblioteca Virtual em Saúde (2021). Conheça a BVS. https://bvsms.saude.gov.br/.

Bortoluzzi, M. M., Schmitt, V. \& Mazur, C. E. (2020). Efeito fitoterápico de plantas medicinais sobre a ansiedade: uma breve revisão. Research, Society and Development, 9(1), p. e02911504-e02911504, 2020.

Carneiro, F. M., Silva, M. J. P., Borges, L. L., Albernaz, L. C. \& Costa, J. D. P (2014). Tendências dos estudos com plantas medicinais no Brasil. Revista Sapiência: sociedade, saberes e práticas educacionais, 3(2), 44-75.

Carvalho, L. G., Leite, S. C. \& Costa, D. A. F. (2021). Principais fitoterápicos e demais medicamentos utilizados no tratamento de ansiedade e depressão. Revista de Casos e Consultoria, 12(1), e25178-e25178.

Cases, J., Ibarra, A., Feuillère, N., Roller, M \& Samir, G. S. (2011). Pilot trial of Melissa officinalis L. leaf extract in the treatment of volunteers suffering from mild-to-moderate anxiety disorders and sleep disturbances. Med J Nutrition Metab, 4(3), 211-218.

Castro, M. R., \& Figueiredo, F. F. (2019). Saberes tradicionais, biodiversidade, práticas integrativas e complementares: o uso de plantas medicinais no SUS. Revista Brasileira de Geografia Médica e da Saúde, 15(31), 56-70.

Cury, A. J. (2017). Ansiedade: como enfrentar o mal do século. Saraiva Educação SA.

Donelli, T. M. S., Chemello, M. R. \& Levandowski, D. C. (2017). Ansiedade materna e maternidade: Revisão crítica da literatura. Interação em Psicologia, 21(1), p. 78-89.

Gotlib, I. H., Joormann, J. (2010). Cognição e depressão: estado atual e direções futuras. Revisão anual de psicologia clínica, 6, $285-312$.

Jesus, J. J. M., \& Oliveira, L. S. (2021). Utilização etnobotânica da espécie medicinal melissa Officinalis L. para o tratamento da ansiedade. Revista IberoAmericana de Humanidades, Ciências e Educação, 7(9), 1078-1089.

Klemow, K. M., Bartlow, A., Crawford, J., Kocher, N., Shah, J., \& Ritsick, M. (2011). Medical attributes of St. John's wort (Hypericum perforatum). Herbal medicine: biomolecular and clinical aspects, 211-237.

Köche, J. C. (2016). Fundamentos de metodologia científica. Editora Vozes. 
Kyrou, I., Christou, A., Panagiotakos, D., Stefanaki, C., Skenderi, K., Katsana, K., \& Tsigos, C. (2017). Effects of a hops (Humulus lupulus L.) dry extract supplement on self-reported depression, anxiety and stress levels in apparently healthy young adults: a randomized, placebo-controlled, double-blind, crossover pilot study. Hormones, 16(2), 171-180.

Lakatos, E. M., \& Marconi, M. D. A. (1996). Técnicas de pesquisa. Atlas, 205.

Lima, S. S., Filho, R. O. L., \& Oliveira, G. L. (2019). Aspectos farmacológicos da Matricaria recutita (camomila) no tratamento do transtorno de ansiedade generalizada e sintomas depressivos. Visão Acadêmica, 20(2), 59-67.

Lopes, M. W., Tiyo, R., Arantes, V. P. (2017). Utilização de Passiflora incarnata no tratamento da ansiedade. Revista Uningá Review, 29(2), 81-86.

Mattos, G., Camargo, A., Sousa, C. A. D., \& Zeni, A. L. B. (2018). Plantas medicinais e fitoterápicos na Atenção Primária em Saúde: percepção dos profissionais. Ciência \& Saúde Coletiva, 23, 3735-3744.

Moreira, M. A., \& Rizzatti, I. M. (2020). Pesquisa em ensino. Revista Internacional de Pesquisa em Didática das Ciências e Matemática, 1, e020007e020007.

Ng, Q. X., Venkatanarayanan, N. \& Ho, C. Y. X. (2017). Clinical use of Hypericum perforatum (St John's wort) in depression: A meta-analysis. Journal of Affective Disorders. Jornal de transtornos afetivos, 210, p. 211-221.

Oliveira, A. C. D., Barreto, T. O. S., \& Neto, J. F. A. (2018). Interação de plantas medicinais com anti-hipertensivos. Revista Ibero-Americana de Humanidades, Ciências e Educação, 7(5), 368-393.

Oliveira, L. M., Filho, A. C. P. M., \& Porfiro, C. A. (2020). Uso da Passiflora incarnata L. no tratamento alternativo do transtorno de ansiedade generalizada. Research, Society and Development, 9(11), e2349119487-e2349119487.

Orhan, I. E (2018). Phytochemical and Pharmacological Activity Profile of Crataegus oxyacantha L. (Hawthorn) - A Cardiotonic Herb Current medicinal chemistry, 25(37), 4854-4865.

Pavanelli, A. S. (2021). Fitoterápicos no controle da depressão e ansiedade.

Pereira, A. S., Shitsuka, D. M., Parreira, F. J., \& Shitsuka, R. (2018). Metodologia da pesquisa científica.

Santos, R., Guimarães, G. P., Nobre, M. S. C. \& Portela, A. S. (2011). Análise sobre a fitoterapia como prática integrativa no Sistema Único de Saúde. Revista brasileira de plantas medicinais, 13, 486-491.

Pereira, L. S., Alencar, A. A., Medeiros, J. P. \& Custódio, L. L. P. (2018). Propriedade ansiolítica da Crataegus oxyacantha: uma breve revisão. Archives of Health Investigation, 7(1).

Silva, M. C., Souza, N. B., Rocha, T. S., Paixão, J. A., \& Alcantara, A. M. C. M. (2021). Utilização da Piper Methysticum (1.) e Passiflora Incarnata (1.) no tratamento de transtorno de ansiedade generalizada. Revista Ibero-Americana de Humanidades, Ciências e Educação, 7(4), 959-973.

Silva, M. J., Gomes, M. L. B., \& Siqueira, L. P. (2021). Alternative treatment for anxiety based on the medicinal plant Melissa Officinalis (lemon balm) - a literature review. Research, Society and Development, 10(14), e532101422349.

Silva, S. T. \& Silva, J. E. S. (2021). Benefícios das plantas medicinais no tratamento da ansiedade e depressão. In: Trajetória e Pesquisas nas Ciências Farmacêuticas, 1(1), 1-388-416.

Zirak, N., Shafiee, M., Soltani, G., Mirzaei, M., \& Sahebkar, A. (2019). Hypericum perforatum in the treatment of psychiatric and neurodegenerative disorders: Current evidence and potential mechanisms of action. Journal of cellular physiology, 234(6), 8496-8508. 